\title{
ULTRASONIC ATTENUATION IN BINARY ALLOYS
}

\author{
S.K. Kor and G. PANDEY \\ Department of Physics, University of Allahabad, Allahabad-211 002, India
}

(Received August 27, 1997; revised version December 8, 1997)

\begin{abstract}
An attempt was made to study the ultrasonic attenuation in $\mathrm{Cu}-\mathrm{Zn}$, $\mathrm{Ag}-\mathrm{Cd}, \mathrm{Ag}-\mathrm{Mg}$ and $\mathrm{Ag}-\mathrm{Zn}$ alloys, due to phonon-phonon interaction at $300 \mathrm{~K}$. The Morse potential was used to evaluate the second and third order elastic constants. How far the neighbouring atoms are effective and contributing to ultrasonic attenuation was studied. It is concluded that most of the ultrasonic attenuation is covered by the atoms from 1st to 8th shell. The contribution to ultrasonic attenuation due to 9 th shell to 12 th shell is very small. It is also concluded that alloys behave more or less in the same manner as metals.
\end{abstract}

PACS numbers: $62.20 . D c, 62.65 .+k, 62.80 .+f$

\section{Introduction}

Ultrasonics is a versatile tool for studying the properties of solids [1], liquids [2] and liquid crystals [3]. Extensive study has been made by us in solids, liquids, and liquid crystals [4-6].

Several potentials [7-9] have been used to study the second order elastic constants (SOEC) and third order elastic constants (TOEC) and subsequently the ultrasonic attenuation due to phonon-phonon ( $\mathrm{p}-\mathrm{p})$ interaction at higher temperature and due to electron-phonon $(e-p)$ interaction below $80 \mathrm{~K}$. The Morse potential explains most of the physical properties in metals, although there are better and accurate methods [10] to study the SOEC and TOEC. The Morse potential with some approximation has several advantages, although it is true that the effective pair potential is not so simple as the Morse potential for the study of physical properties of metallic crystals. The advantages of using Morse potential being, very simple, its overall quantitative agreement between theoretical and experimental results are good and give definite idea about the interaction of atoms in different shells and also that the different Morse parameters are obtained from the experimental values of lattice parameter, bulk modulus and cohesive energy and thus the results are reliable.

With minor modifications the Morse potential is quite useful in describing the properties of metallic solid solutions [11]. The binary alloys are usually treated by the quasi-chemical approach. Here, we have used the simple average Morse 
potential $\phi(r)$, by which the interaction between any atoms in the alloy may be represented. The potential is obviously fictitious and is given the name virtual Morse potential (VMP) [11]. The parameters in VMP are determined as given above, in the same manner as the usual Morse potential.

In the present study, VMP was used to evaluate SOEC and TOEC and the contribution of different shells to it and then evaluation of ultrasonic attenuation at $300 \mathrm{~K}$ due to $\mathrm{p}-\mathrm{p}$ interaction in several alloys viz. $\mathrm{Cu}-\mathrm{Zn}, \mathrm{Ag}-\mathrm{Mg}, \mathrm{Ag}-\mathrm{Cd}$ and $\mathrm{Ag}-\mathrm{Zn}$, is made, for different composition of one metal into the other. A consideration was made for phase transition of different mixtures studied.

\section{Theory}

\subsection{Determination of virtual Morse potential parameters}

The interaction energy according to VMP is given by

$$
\phi\left(r_{i j}\right)=D\left[\mathrm{e}^{-2 \alpha\left(r_{i j}-r_{0}\right)}-2 \mathrm{e}^{-\alpha\left(r_{i j}-r_{0}\right)}\right] .
$$

Here $D, \alpha, r_{0}$ are VMP parameters: $D=\phi\left(r_{0}\right)$ is a cohesive energy or sublimation energy at zero pressure and temperature, $\alpha$ is a constant with dimensions of reciprocal distance, which depends on the hardness of the potential, $r_{0}$ is the distance of $i$-th atom from the origin $(0,0,0)$, and $r_{i j}$ is the distance between any two atoms in the alloy. These parameters at each alloy composition can be determined using the following conditions/equations. The total energy of a crystal containing $N$ atoms is given by

$$
E\left(a_{0}\right)=\Phi=\frac{1}{2} N D \sum_{j=1}^{J} \phi\left(r_{i j}\right)
$$

which is also obtained as

$$
E\left(a_{0}\right)=S_{\mathrm{A}}\left(1-X_{\mathrm{B}}\right)+S_{\mathrm{B}} X_{\mathrm{B}}+\Delta H .
$$

Here $S_{\mathrm{A}}$ and $S_{\mathrm{B}}$ are sublimation energies of $\mathrm{A}$ and $\mathrm{B}$ [12], $X_{\mathrm{B}}$ is the atomic fraction of $\mathrm{B}$ in the alloy $\mathrm{AB}, \Delta H$ is the heat of mixing [13], and $a_{0}$ is the lattice parameter [14].

$$
\begin{aligned}
& \left(\frac{\mathrm{d} E}{\mathrm{~d} a}\right)_{a=a_{0}}=0, \\
& B=\frac{a_{0}}{9 V_{0}}\left(\frac{\mathrm{d}^{2} E}{\mathrm{~d} a^{2}}\right)_{a \rightarrow a_{0}} .
\end{aligned}
$$

Here $B$ is a bulk modulus [12].

\subsection{Elastic constants}

The $\alpha$-phase of the alloys studied are examples of face centered cubic (fcc) crystals. For a cubic crystal, there are three independent SOEC $\left(C_{11}, C_{12}, C_{44}\right)$ and six independent TOEC $\left(C_{111}, C_{112}, C_{123}, C_{456}, C_{144}, C_{166}\right)$. The expression for SOEC's is as follows: 


$$
\begin{aligned}
C_{11} & =\frac{2 D a^{2} \alpha^{2} \beta^{2}}{V_{0}} \sum \frac{m_{j} \mathrm{e}^{-2 \alpha a M_{j}}}{M_{j}}-\frac{2 D a^{2} \alpha^{2} \beta}{V_{0}} \sum \frac{m_{j}^{4} \mathrm{e}^{-\alpha a M_{j}}}{M_{j}^{2}} \\
& +\frac{D a \alpha \beta^{2}}{V_{0}} \sum \frac{m_{j}^{4} \mathrm{e}^{-2 \alpha a M_{j}}}{M_{j}^{3}}-\frac{D a \alpha \beta}{V_{0}} \sum \frac{m_{j}^{4} \mathrm{e}^{-\alpha a M_{j}}}{M_{j}^{3}}
\end{aligned}
$$

where $\beta=\mathrm{e}^{\alpha r_{0}}$ and $r_{j}=\left[m_{j}^{2}+n_{j}^{2}+l_{j}^{2}\right]^{1 / 2} a=M_{j} a$ and $V_{0}$ is the volume of fcc lattice.

The expressions for $C_{12}$ and $C_{44}$ are obtained by replacing $m_{j}^{4}$ by $m_{j}^{2} n_{j}^{2}$.

The expression for TOEC's is as follows:

$$
\begin{aligned}
C_{111} & =\frac{4 D a^{3} \alpha^{3} \beta^{2}}{V_{0}} \sum \frac{m_{j}^{6} \mathrm{e}^{-2 \alpha a M_{j}}}{M_{j}^{3}}-\frac{6 D a^{2} \alpha^{2} \beta}{V_{0}} \sum \frac{m_{j}^{6} \mathrm{e}^{-2 \alpha a M_{j}}}{M_{j}^{4}} \\
& -\frac{3 D a \alpha \beta^{2}}{V_{0}} \sum \frac{m_{j}^{6} \mathrm{e}^{-2 \alpha a M_{j}}}{M_{j}^{5}}+\frac{D a^{3} \alpha^{3} \beta}{V_{0}} \sum \frac{m_{j}^{6} \mathrm{e}^{-\alpha a M_{j}}}{M_{j}^{3}} \\
& +\frac{3 D a^{2} \alpha^{2} \beta}{V_{0}} \sum \frac{m_{j}^{6} \mathrm{e}^{-2 \alpha a M_{j}}}{M_{j}^{4}}+\frac{3 D a \alpha \beta}{V_{0}} \sum \frac{m_{j}^{6} \mathrm{e}^{-\alpha a M_{j}}}{M_{j}^{5}} .
\end{aligned}
$$

The expressions for $C_{112}$ and $C_{166}$ are obtained by replacing $m_{j}^{6}$ by $m_{j}^{4} n_{j}^{2}$ and $C_{123}, C_{456}$ and $C_{144}$ are obtained by replacing $m_{j}^{6}$ by $m_{j}^{2} n_{j}^{2} l_{j}^{2}$ in all the summations.

Here $D$ is the dissociation energy, $\alpha$ is a parameter related to the hardness of the potential, $r_{0}$ is the separation of atoms from minimum potential, they are all Morse potential parameters which are determined from the experimental values of lattice parameter $\left(a_{0}\right)$, bulk modulus $(B)$ and cohesive energy $(E)$.

\subsection{Ultrasonic attenuation due to $p-p$ interaction}

Akhieser [15] first proposed the ultrasonic attenuation due to $p-p$ interaction, which was modified by Bömmel and Dransfeld [16] and finally by Mason [17]. Presently we have used the modified form of Mason [17] which is used [5, 18] for determining $\left(\alpha / f^{2}\right)_{l}$ and $\left(\alpha / f^{2}\right)_{s}$ for longitudinal and shear waves respectively, using non-linearity constant which is related to Grüneisen constant, which in turn is related to SOEC and TOEC.

\section{Evaluation}

Using known experimental values of lattice parameter [14], bulk modulus [12] and cohesive energy [12], $D, \alpha$, and $r_{0}$ are calculated using a small computer program developed by us. These values of $D, \alpha$ and $r_{0}$ (Table I) were used to evaluate SOEC and TOEC for different shells, starting from 1st shell to 12th shell. The SOEC and TOEC thus evaluated are then used to obtain Grüneisen constants and finally the ultrasonic attenuation $[4,18]$. 


\section{TABLE I}

Values of $D, \alpha, r_{0}, a_{0}$ used to calculate the SOEC's and TOEC's for different alloy compositions.

\begin{tabular}{r|c|c|c|c}
\hline \hline$X$ & $D[\mathrm{eV}]$ & $\alpha[1 / \AA]$ & $r_{0}[\AA]$ & $a_{0}[\AA]$ \\
\hline \multicolumn{5}{c}{$\mathrm{Cu}-\mathrm{Zn}, X=X_{\mathrm{Zn}}$} \\
\hline 0.00 & 0.3282 & 1.3123 & 2.8985 & 1.8070 \\
3.99 & 0.3151 & 1.3295 & 2.9249 & 1.8339 \\
4.47 & 0.3135 & 1.3316 & 2.9281 & 1.8371 \\
8.82 & 0.2991 & 1.3506 & 2.9572 & 1.8667 \\
16.70 & 0.2734 & 1.3844 & 3.0091 & 1.9194 \\
22.20 & 0.2554 & 1.4082 & 3.0455 & 1.9564 \\
\hline \multicolumn{5}{c}{$\mathrm{Ag}-\mathrm{Zn}, X=X_{\mathrm{Zn}}$} \\
\hline 0.00 & 0.3253 & 1.3535 & 3.1300 & 2.0390 \\
3.90 & 0.3126 & 1.3687 & 3.1468 & 2.0562 \\
5.69 & 0.3068 & 1.3757 & 3.1545 & 2.0641 \\
\hline \multicolumn{5}{c}{$\mathrm{Ag}-\mathrm{Mg}, X=X_{\mathrm{Mg}}$} \\
\hline 12.32 & 0.2852 & 1.3458 & 3.2180 & 2.1093 \\
25.98 & 0.2408 & 1.3373 & 3.3157 & 2.1873 \\
\hline \multicolumn{5}{c}{$\mathrm{Ag}-\mathrm{Cd}, X=X_{\mathrm{Cd}}$} \\
\hline 1.29 & 0.3211 & 1.3589 & 3.1413 & 2.0489 \\
1.84 & 0.3193 & 1.3612 & 3.1461 & 2.0532 \\
\multicolumn{5}{c}{}
\end{tabular}

\section{Results and discussion}

The SOEC and TOEC are evaluated for atoms from 1st shell to 12th shell and large amount of data are obtained. Some of the results are presented here. For $\mathrm{Cu}-\mathrm{Zn}$ and $\mathrm{Ag}-\mathrm{Cd}, C_{11}$ and $C_{12}$ for interaction of atoms for up to 6, 8, 10, and 12 shells are presented in Table II along with the experimental values [11]. It is clear that the results are in good agreement showing the validity of the work. For $\mathrm{Ag}-\mathrm{Mg}$ and $\mathrm{Ag}-\mathrm{Zn}$ the $C_{11}$ and $C_{44}$ values are presented in Table III along with the experimental values [11], showing good agreement.

Table IV presents all the values of ultrasonic attenuation evaluated in the present work for different compositions of one alloying metal into the other. Although no experimental values of ultrasonic attenuation are available, we tried to justify our work by extrapolating the results, so as to obtain the ultrasonic attenuation in pure $\mathrm{Cu}$ and the value so obtained is compared with the experimental value available [19]. The result is in very good agreement and it may be concluded that the method applied is reliable. 
TABLE II

SOEC $\left(C_{11}\right.$ and $\left.C_{12}\right)$ as evaluated for interaction of atoms in different shells from 6 th to 12 th shell as compared to experimental values (in $10^{12} \mathrm{dyn} / \mathrm{cm}^{2}$ ).

\begin{tabular}{r|c|c|c|c|c|c|c|c|c|c|c}
\hline \hline \multirow{2}{*}{$X$} & \multicolumn{2}{|c|}{6 th } & \multicolumn{2}{c|}{8 th } & \multicolumn{2}{c|}{10 th } & \multicolumn{2}{c|}{12 th } & \multicolumn{2}{c}{ Expt. value $^{\mathrm{a}}$} \\
\cline { 2 - 11 } & $C_{11}$ & $C_{12}$ & $C_{11}$ & $C_{12}$ & $C_{11}$ & $C_{12}$ & $C_{11}$ & $C_{12}$ & $C_{11}$ & $C_{12}$ \\
\hline \multicolumn{8}{c}{$\mathrm{Cu}-\mathrm{Zn}, X=X_{\mathrm{zn}}$} \\
\hline 0.00 & 1.939 & 1.260 & 1.715 & 1.163 & 1.634 & 1.145 & 1.591 & 1.128 & 1.792 & 1.262 \\
3.99 & 1.891 & 1.217 & 1.707 & 1.138 & 1.644 & 1.124 & 1.610 & 1.110 & 1.633 & 1.177 \\
4.47 & 1.885 & 1.212 & 1.705 & 1.134 & 1.644 & 1.121 & 1.610 & 1.108 & 1.634 & 1.192 \\
8.82 & 1.823 & 1.162 & 1.680 & 1.099 & 1.632 & 1.089 & 1.606 & 1.079 & 1.571 & 1.137 \\
10.70 & 1.693 & 1.064 & 1.598 & 1.022 & 1.569 & 1.016 & 1.554 & 1.010 & 1.499 & 1.097 \\
22.20 & 1.590 & 0.990 & 1.520 & 0.959 & 1.500 & 0.954 & 1.488 & 0.950 & 1.447 & 1.071 \\
\hline \multicolumn{8}{|c|}{$\mathrm{Ag}-\mathrm{Cd}, X=X_{\mathrm{Cd}}$} \\
\hline 1.29 & 1.415 & 0.919 & 1.346 & 0.888 & 1.326 & 0.884 & 1.316 & 0.880 & 1.228 & 0.925 \\
1.84 & 1.412 & 0.915 & 1.345 & 0.885 & 1.326 & 0.881 & 1.316 & 0.887 & 1.216 & 0.913 \\
\hline
\end{tabular}

${ }^{a}$ Ref. [11].

TABLE III

SOEC $\left(C_{11}\right.$ and $\left.C_{44}\right)$ as evaluated for interaction of atoms in different shells from 6 th to 12 th shell as compared to experimental values (in $10^{12} \mathrm{dyn} / \mathrm{cm}^{2}$ ).

\begin{tabular}{c|c|c|c|c|c|c|c|c|c|c|c}
\hline \hline$X$ & \multicolumn{2}{|c|}{6 th } & \multicolumn{2}{c|}{8 th } & \multicolumn{2}{c|}{10 th } & \multicolumn{2}{c|}{12 th } & \multicolumn{2}{c}{ Expt. value } \\
\cline { 2 - 10 } & $C_{11}$ & $C_{44}$ & $C_{11}$ & $C_{44}$ & $C_{11}$ & $C_{44}$ & $C_{11}$ & $C_{44}$ & $C_{11}$ & $C_{44}$ \\
\hline \multicolumn{8}{c}{$\mathrm{Ag}-\mathrm{Zn}, X=X_{\mathrm{Zn}}$} \\
\hline 0.00 & 1.422 & 0.927 & 1.348 & 0.895 & 1.326 & 0.890 & 1.315 & 0.886 & 1.240 & 0.934 \\
3.90 & 1.394 & 0.901 & 1.331 & 0.873 & 1.313 & 0.869 & 1.304 & 0.866 & 1.239 & 0.916 \\
5.69 & 1.379 & 0.888 & 1.321 & 0.862 & 1.304 & 0.859 & 1.296 & 0.856 & 1.230 & 0.933 \\
\hline \multicolumn{10}{c}{$\mathrm{Ag}-\mathrm{Mg}, X=X_{\mathrm{Mg}}$} \\
\hline 12.32 & 1.163 & 0.754 & 1.113 & 0.732 & 1.098 & 0.729 & 1.091 & 0.726 & 1.198 & 0.898 \\
25.98 & 0.908 & 0.585 & 0.876 & 0.571 & 0.867 & 0.569 & 0.863 & 0.568 & 1.159 & 0.866 \\
\hline
\end{tabular}

${ }^{a}$ Ref. [11]:

\section{Conclusions}

The following conclusions may be drawn from the present study:

(1) The elastic constants obtained are in good agreement with available experimental values.

(2) Ultrasonic attenuation is dependent on the number of atoms in a shell and on the distances of atoms from the central atom.

(3) The ultrasonic attenuation is dependent on the number of shells included in the evaluation.

(4) The contribution to ultrasonic attenuation due to interaction of atoms above 8 th shell is very small but not negligible.

(5) Alloys behave more or less in the same manner as the metals. 


\section{TABLE IV}

\begin{tabular}{|c|c|c|c|c|}
\hline \multirow[t]{2}{*}{$X$} & \multicolumn{4}{|c|}{ Up to no. of shells } \\
\hline & 6 th & 8th & 10th & $12 \mathrm{th}$ \\
\hline \multicolumn{5}{|c|}{$\mathrm{Cu}-\mathrm{Zn}, X=X_{\mathrm{Zn}}$} \\
\hline 0.00 & 0.31 & 0.48 & 0.58 & 0.45 \\
\hline 3.99 & 0.27 & 0.37 & 0.42 & 0.45 \\
\hline 4.47 & 0.26 & 0.36 & 0.41 & 0.44 \\
\hline 8.82 & 0.23 & 0.29 & 0.31 & 0.33 \\
\hline 10.70 & 0.19 & 0.22 & 0.23 & 0.23 \\
\hline 22.20 & 0.17 & 0.19 & 0.19 & 0.19 \\
\hline \multicolumn{5}{|c|}{$\mathrm{Ag}-\mathrm{Zn}, X=X_{\mathrm{Zn}}$} \\
\hline 0.00 & 1.23 & 1.44 & 1.50 & 1.54 \\
\hline 3.90 & 1.03 & 1.17 & 1.21 & 1.23 \\
\hline 5.69 & 0.95 & 1.07 & 1.10 & 1.12 \\
\hline \multicolumn{5}{|c|}{$\mathrm{Ag}-\mathrm{Cd}, X=X_{\mathrm{Cd}}$} \\
\hline 1.29 & 1.13 & 1.31 & 1.36 & 1.39 \\
\hline 1.84 & 1.13 & 1.30 & 1.35 & 1.38 \\
\hline \multicolumn{5}{|c|}{$\mathrm{Ag}-\mathrm{Mg}, X=X_{\mathrm{Mg}}$} \\
\hline 12.32 & 1.46 & 1.65 & 1.71 & 1.73 \\
\hline 25.98 & 1.83 & 2.01 & 2.06 & 2.08 \\
\hline
\end{tabular}

(6) The method applied may not be so rigorous as other methods, but the method is simple and the results obtained are satisfactory. The method also gives a clear physical picture of contribution to ultrasonic attenuation by different shells.

\section{Acknowledgment}

The authors would like to thank the University Grants Commission (India) for financial assistance and to the Head of the Department of Physics, for providing computer and other facilities in the department.

\section{References}

[1] W.P. Mason, Physical Acoustics, Academic Press, New York 1965, Vol. 3B.

[2] W.P. Mason, in Ref. [1], Vol. 2A.

[3] K. Miyano, J.K. Kelterson, Physical Acoustics A 6, 2401 (1972).

[4] S.K. Kor, P.K. Mishra, U.S. Tandon, Solid State Commun. 15, 499 (1974).

[5] S.K. Kor, S.C. Deorani, Phys. Rev. Lett. 27, 242 (1971).

[6] S.K. Kor, S.K. Pandey, J. Chem. Phys. 64, 1333 (1976). 
[7] M. Born, J.B. Mayer, Z. Phys. 75, 1 (1931).

[8] B.R.K. Gupta, U. Kewald, D.B. Ghate, J. Phys. Condens. Matter 4, 6879 (1992).

[9] L.A. Garifalco, V.G. Weiser, Phys. Rev. 114, 687 (1959).

[10] J.C. Boettger, Phys. Rev. B, Condens. Matter 55, 17, 11202 (1997).

[11] Y. Yamamoto, M. Doyama, J. Phys. Chem. Solids 35, 759 (1974).

[12] American Institute of Physics Handbook, Ed. D.E. Gray, McGraw-Hill, New York 1963.

[13] R. Hultgren, R.L. Orr, P.D. Anderson, K.K. Kelly, Selected Values of Thermodynamic Values of Metals and Alloys, Wiley, New York 1963.

[14] W.B. Peason, A Handbook of Lattice Spacings and Structures of Metals and Alloys, Pergamon Press, Oxford 1958.

[15] A. Akhiezer, J. Phys. (USSR) 1, 227 (1939).

[16] H.E. Bömmel, K. Dransfeld, Phys. Rev. 117, 245 (1960).

[17] W.P. Mason, Piezoelectric Crystals and Their Applications to Ultrasonics, Van Nostrand, Princeton 1960.

[18] S.K. Kor, R.K. Singh, Acta Phys. Pol. A 83, 751 (1993).

[19] W.P. Mason, A. Rosenberg, J. Acoust. Soc. Am. 45, 470 (1969). 